\title{
Circle Actions and Higher Elliptic Genera
}

By

\author{
Yasuhiro HARA*
}

\begin{abstract}
For a manifold with an $S^{1}$-action, we define generalized elliptic genera by using the orbit map and generalize Hirzebruch-Slodowy's formula in [9]. As a result, we have vanishing theorems of higher elliptic genera and higher twisted $\hat{A}$-genera. We also generalize elliptic genera of level $N$ for stable almost complex manifolds and have a similar vanishing theorem.
\end{abstract}

\section{$\S$ 且。 Introduction}

Elliptic genera were introduced by S. Ochanine [13]. The $\hat{A}$-genus and the signature are special cases of elliptic genera. We know many results concerning the $\hat{A}$-genus and the signature which are related with group actions (cf. [10]). Some of these results were extended to the case of elliptic genera. For example, the vanishing theorem of the $\hat{A}$-genus [2] was extended to the rigidity theorem of elliptic genera by Bott-Taubes [4]. For a manifold with an involution, Hirzebruch and Slodowy [9] proved the relation between the elliptic genera of the manifold and the elliptic genera of the fixed point set, which is a generalization of an old formura for the signature. Moreover in [8], Hirzebruch defined elliptic genera of level $N$ for almost complex manifolds and proved the rigidity of those genera.

On the other hand, the vanishing theorem of the $\hat{A}$-genus above was generalized to the vanishing theorem of the higher $\hat{A}$-genus by Browder-Hsiang [6]. In their proof, they first generalized the $\hat{A}$-genus by using an orbit map and proved the vanishing of the generalized $\hat{A}$-genus by using the equivariant surgery. $\mathrm{H}-\mathrm{T}$. $\mathrm{Ku}$ and $\mathrm{M}-\mathrm{C} . \mathrm{Ku}$ [11] generalized the signature in a similar way and proved the generalized G-signature theorem.

In this paper, we first define generalized elliptic genera in a similar way and generalize the Hirzebruch-Slodowy's theorem above. Consequently, we have some vanishing theorems of higher elliptic genera and higher twisted $\hat{A}$-genera. After that, we generalize the elliptic genera of level $N$ for stable almost complex manifolds and have a similar vanishing theorem.

The author wishes to thank Professor K. Kawakubo for his useful advice.

Communicated by S. Mori, December 20, 1993.

1991 Mathematics Subject Classifications: 57R20, 57S 15.

* Department of Mathematics, Osaka University, Toyonaka Osaka 560, Japan 


\section{§2. Elliptic Genera}

Let $\Omega^{S O}$ be the oriented cobordism ring and $\Lambda$ any commutative $\mathbb{Q}$-algebra with unit. A genus is a ring homomorphism

$$
\varphi: \Omega^{S O} \rightarrow \Lambda
$$

with $\varphi(1)=1$. Since $\Omega_{1}^{S O} \otimes \mathbb{Q}=\mathbf{Q}\left[\left[\mathbf{C} P^{2}\right],\left[\mathbf{C} P^{4}\right],\left[\mathbf{C} P^{6}\right], \ldots\right], \varphi$ is determined by the logarithm

$$
g(x)=\sum_{n \geq 0} \frac{\varphi\left(\mathbf{C} P^{2 n}\right)}{2 n+1} x^{2 n+1}
$$

Following Ochanine [14], we call $\varphi$ an elliptic genus if $g(x)$ has the form

$$
g(x)=\int_{0}^{1} \frac{d t}{\sqrt{1-2 \delta t^{2}+\varepsilon t^{4}}}
$$

with $\delta, \varepsilon \in \Lambda$. We remark that for any elliptic genus $\varphi$ one has $\delta=\varphi\left(\mathbf{C} P^{2}\right)$, $\varepsilon=\varphi\left(\mathbf{H} P^{2}\right)$.

Let $E$ be a real vector bundle over $X$. We write $\Lambda^{\prime}(E)$ and $S^{\prime}(E)$ for the exterior and the symmetric powers of $E$ respectively, and set

$$
\begin{aligned}
& \Lambda_{t}(E)=\sum_{t \geq 0} \Lambda^{\prime}(E) t^{\prime} \\
& S_{t}(E)=\sum_{t \geq 0} S^{\prime}(E) t^{\prime} .
\end{aligned}
$$

Define

$$
\mathscr{K}_{q}(E)=\bigotimes_{n \geq 0}\left(\Lambda_{q^{\prime \prime}}(E) \otimes S_{q^{\prime \prime}}(E)\right)
$$

and

$$
\Theta_{q}(E)=\bigotimes_{n \geq 0}\left(\Lambda_{-q^{2 n-1}}(E) \otimes S_{q^{2 n}}(E)\right) .
$$

Then $\mathscr{K}_{q}(E)$ and $\Theta_{q}(E)$ are formal power series in q with coefficients in $K O(X)$. Moreover

$$
\mathscr{R}_{q}(E \oplus F)=\mathscr{\mathscr { R } _ { q }}(E) \cdot \mathscr{R}_{q}(F)
$$

and

$$
\Theta_{q}(E \oplus F)=\Theta_{q}(E) \Theta_{q}(F),
$$

hence $\mathscr{R}_{q}$ and $\Theta_{q}$ can be extended to $K O(X)$.

For a closed $n$-dimensional oriented smooth manifold $M$, we define

$$
\Phi_{1}(M)=\left\langle\hat{L}(M) \operatorname{ch}\left(\mathscr{Y}_{\varphi}(T(M)-[n]) \otimes \mathbb{C}\right),[M]\right\rangle
$$


and

$$
\Phi_{2}(M)=\left\langle\hat{A}(M) \operatorname{ch}\left(\Theta_{q}(T(M)-[n]) \otimes \mathbf{C}\right),[M]\right\rangle,
$$

where $T(M)$ is the tangent bundle of $M, \hat{L}$ and $\hat{A}$ are multiplicative sequences for characteristic power series $x / 2 \tanh (x / 2)$ and $x / 2 \sinh (x / 2)$ respectively. Note that $\Phi_{1}$ and $\Phi_{2}$ are genera with respect to the characteristic power series $Q_{1}(x)$ and $Q_{2}(x)$ respectively, where

$$
\begin{gathered}
Q_{1}(x)=\frac{x / 2}{\tanh (x / 2)} \prod_{n=1}^{\infty} \frac{\left(1+q^{n} e^{-1}\right)\left(1+q^{n} e^{1}\right) /\left(1+q^{n}\right)^{2}}{\left(1-q^{n} e^{-1}\right)\left(1-q^{n} e^{x}\right) /\left(1-q^{n}\right)^{2}} \\
Q_{2}(x)=\frac{x / 2}{\sinh (x / 2)} \prod_{n=1}^{\infty} \frac{\left(1-q^{2 n-1} e^{-1}\right)\left(1-q^{2 n-1} e^{1}\right) /\left(1-q^{2 n-1}\right)^{2}}{\left(1-q^{2 n} e^{-1}\right)\left(1-q^{2 n} e^{1}\right) /\left(1-q^{2 n}\right)^{2}} .
\end{gathered}
$$

We now recall the following theorem due to D. Zagier.

Theorem 2.1 ([14], cf. [7]). (i) $\Phi_{1}$ is an elliptic genus with

$$
\begin{aligned}
& \delta=\frac{1}{4}+6 \sum_{n=1}^{\infty}\left(\sum_{\substack{d \mid n \\
d n d d}} d\right) q^{n} \\
& \varepsilon=\frac{1}{16} \prod_{n=1}^{\infty}\left(\frac{1-q^{n}}{1+q^{n}}\right)^{8} .
\end{aligned}
$$

(ii) $\Phi_{2}$ is an elliptic genus with

$$
\begin{aligned}
& \tilde{\delta}=-\frac{1}{8}-3 \sum_{n=1}^{\infty}\left(\sum_{\substack{d|n \\
d| n d l}} d\right) q^{n} \\
& \tilde{\varepsilon}=\sum_{n=1}^{\infty}\left(\sum_{\substack{d \mid n \\
\text { uld oudd }}} d^{3}\right) q^{n} .
\end{aligned}
$$

It is known that these genera have the modular properties. If we put $q=e^{2 \pi \tau \tau}$ with $\tau \in \mathfrak{h}$ (upper half plane), then the values of these genera are modular forms on $\Gamma_{0}(2)=\left\{\left(\begin{array}{ll}a & b \\ c & d\end{array}\right) \in S L_{2}(\mathbf{Z}) \mid c \equiv 0(\bmod 2)\right\}$. Let $M_{h}(2)$ denote the complex vector space of all modular forms of weight $k$ on $\Gamma_{0}(2)$. Then $\delta, \tilde{\delta} \in M_{2}(2)$ and $\varepsilon, \tilde{\varepsilon} \in$ $M_{4}(2)$. Moreover for the graded ring $M_{1}(2)=\bigoplus_{k \in \mathbf{Z}} M_{k}(2)$, we have

$$
M_{\cdot}(2)=\mathbf{C}[\delta, \varepsilon] .
$$

In particular, $\delta$ and $\varepsilon$ are algebraically independent. 


\section{§3. Circle Actions amd Higher Elliptic Gemera}

In this section, we consider manifolds with circle actions. We will assume from now on (unless stated explicitly otherwise) that all $S^{1}$-actions are smooth and effective. Hirzebruch and Slodowy proved the following theorem.

Theorem 3.1 ([9], cfi。 [3])。 Let $M$ be a 4k-dimensional closed spin manifold with an $S^{1}$-action. Let $M^{I}$ be the fixed point set of the involution $I$ which is induced by the $S^{1}$-action. Let $F_{\lambda}$ be a connected component of $M^{l}$, and $d_{\lambda}$ the codimension of $F_{\lambda}$ in $M$. Then,

$$
\Phi_{1}(M)=\sum_{\lambda} \Phi_{1}\left(F_{\lambda} \circ F_{\lambda}\right) \varepsilon^{d_{\lambda} / 4}
$$

where $F_{\lambda} \circ F_{\lambda}$ is the self-intersection of $F_{\lambda}$ and $\varepsilon$ is as in Theorem 2.1, (i).

For an $m$-dimensional closed oriented smooth manifold $M$ and $z \in H^{\prime}(M ; \mathbb{Q})$, we define

$$
\Phi_{1}(M, z)=\langle\hat{L}(M) \operatorname{ch}(\mathscr{K}(T(M)-[m]) \otimes \mathbb{C}) \cup z,[M]\rangle .
$$

We can generalize the theorem above as follows.

Theorem 3.2. Let $M$ be an m-dimensional closed spin manifold with an $S^{1}$-action. Let $I, F_{\lambda}$ and $d_{\lambda}$ be as in Theorem 3.1 and $p: M \rightarrow M / S^{1}$ the orbit map. Then for $x \in H\left(M / S^{1} ; \mathbb{Q}\right)$

$$
\Phi_{1}(M, p x)=\sum_{\lambda} \Phi_{1}\left(F_{\lambda} \circ F_{\lambda}, i_{\lambda} p x\right) \varepsilon^{d_{\lambda} / 4}
$$

where $i_{\lambda}: F_{\lambda} \circ F_{\lambda} \rightarrow M$ is the inclusion.

Proof. Following the proof of [6, Theorem 1.8] (cf. [5]), for any $x \in$ $H^{\prime}\left(M / S^{1} ; \mathbb{Q}\right)$ there exist a transverse framed $S^{1}$-submanifold $N$ of $M \times \mathbb{R}^{h}$ and $c \in \mathbb{Q}-\{0\}$ such that

$$
j[N]=c p \quad x \cap[M]
$$

where $S^{1}$ acts trivially on the $\mathbb{R}^{k}$ and $j: N \rightarrow M \times \mathbb{R}^{k}$ is the inclusion. Then

$$
\begin{aligned}
\Phi_{1}(N) & =\langle j(\hat{L}(M) \operatorname{ch}(\mathscr{R}(T(M)-[m]) \otimes \mathbb{C})),[N]\rangle \\
& =\langle\hat{L}(M) \operatorname{ch}(\mathscr{R}(T(M)-[m]) \otimes \mathbb{C}), j[N]\rangle \\
& =\langle\hat{L}(M) \operatorname{ch}(\mathscr{R}(T(M)-[m]) \otimes \mathbb{C}), c p x \cap[M]\rangle \\
& =c\left\langle\hat{L}(M) \operatorname{ch}(\mathscr{R}(T(M)-[m]) \otimes \mathbb{C}) \cup p^{\prime} x,[M]\right\rangle \\
& =c \Phi_{1}(M, p x) .
\end{aligned}
$$


We put $\tilde{F}_{\lambda}=\left(F_{\lambda} \times \mathbb{R}^{h}\right) \cap N$. We denote the normal bundle of $\tilde{F}_{\lambda}$ in $N$ and the normal bundle of $F_{\lambda}$ in $M$ by $\tilde{v}_{\lambda}$ and $\nu_{\lambda}$ respectively.

Let $j_{\lambda}: \tilde{F}_{\lambda} \rightarrow F_{\lambda} \times \mathbb{R}^{h}$ and $h_{\lambda}: F_{\lambda} \rightarrow M$ be the inclusions. We denote the Euler classes of $\tilde{v}_{\lambda}$ and $v_{\lambda}$ by $e\left(\tilde{v}_{\lambda}\right)$ and $e\left(v_{\lambda}\right)$ respectively. Then

$$
\begin{aligned}
\Phi_{1}\left(\tilde{F}_{\lambda} \circ \tilde{F}_{\lambda}\right) & =\left\langle\hat{L}\left(\tilde{F}_{\lambda}\right) \hat{L}\left(\tilde{v}_{\lambda}\right)^{-1} \operatorname{ch}\left(\mathscr{R}\left(T\left(\tilde{F}_{\lambda}\right)-\tilde{v}_{\lambda}-[m-l]\right) \otimes \mathbb{C}\right) \cup e\left(\tilde{v}_{\lambda}\right),\left[\tilde{F}_{\lambda}\right]\right\rangle \\
& =\left\langle j_{\lambda}\left(\hat{L}\left(F_{\lambda}\right) \hat{L}\left(v_{\lambda}\right)^{-1} \operatorname{ch}\left(\mathscr{R}\left(T\left(F_{\lambda}\right)-v_{\lambda}-[m]\right) \otimes \mathbb{C}\right) \cup e\left(v_{\lambda}\right)\right),\left[\tilde{F}_{\lambda}\right]\right\rangle \\
& =\left\langle\hat{L}\left(F_{\lambda}\right) \hat{L}\left(v_{\lambda}\right)^{-1} \operatorname{ch}\left(\mathscr{R}\left(T\left(F_{\lambda}\right)-v_{\lambda}-[m]\right) \otimes \mathbb{C}\right) \cup e\left(v_{\lambda}\right), j_{\lambda^{\prime}}\left[\tilde{F}_{\lambda}\right]\right\rangle \\
& =\left\langle\hat{L}\left(F_{\lambda}\right) \hat{L}\left(v_{\lambda}\right)^{-1} \operatorname{ch}\left(\mathscr{R}\left(T\left(F_{\lambda}\right)-v_{\lambda}-[m]\right) \otimes \mathbb{C}\right) \cup e\left(v_{\lambda}\right), \operatorname{ch} p^{\prime} p^{\prime}(x) \cap\left[F_{\lambda}\right]\right\rangle \\
& =c\left\langle\hat{L}\left(F_{\lambda}\right) \hat{L}\left(v_{\lambda}\right)^{-1} \operatorname{ch}\left(\mathscr{R}\left(T\left(F_{\lambda}\right)-v_{\lambda}-[m]\right) \otimes \mathbb{C}\right) \cup e\left(v_{\lambda}\right) \cup h_{\lambda}^{\prime} p^{\prime} x,\left[F_{\lambda}\right]\right\rangle \\
& =c \Phi_{1}\left(F_{\lambda} \circ F_{\lambda}, i_{\lambda}^{\prime} p x\right) .
\end{aligned}
$$

If $m-l \not \equiv 0(\bmod 4)$, then $\Phi_{1}\left(M, p^{\prime} x\right)=0$ and $\Phi_{1}\left(F_{\lambda} \circ F_{\lambda}, i_{\lambda}^{\prime} p^{\prime} x\right)=0$ for any $\lambda$. If $m-l \equiv 0(\bmod 4)$, it follows from Hirzebruch-Slodowy's theorem above that

$$
\Phi_{1}(N)=\sum_{\lambda} \Phi_{1}\left(\tilde{F}_{\lambda} \circ \tilde{F}_{\lambda}\right) \varepsilon^{d_{\lambda} / 4}
$$

Therefore

$$
\Phi_{1}(M, p x)=\sum_{\lambda} \Phi_{1}\left(F_{\lambda} \circ F_{\lambda}, i_{\lambda}^{\prime} p x\right) \varepsilon^{d_{\lambda} / 4}
$$

Let $M$ be a closed oriented smooth manifold and $K(\pi, 1)$ an EilenbergMacLane space. For a map $f: M \rightarrow K(\pi, 1)$ and $x \in H^{\prime}(K(\pi, 1) ; \mathbb{Q})$, we call $\Phi_{1}\left(M, f^{\prime} x\right)$ a higher elliptic genus (cf. [12]).

From Theorem 3.2 and [6, Theorem 1.1], we have the following corollary.

Corollary 3.3. Let $M, F_{\lambda}$ and $d_{\lambda}$ be the same as in Theorem 3.2. Suppose that $f: M \rightarrow K(\pi, 1)$ is a map with $f_{1}: \pi_{1}(M) \rightarrow \pi$ surjective and that $\alpha: \pi \rightarrow \pi^{\prime}$ $=\pi / f i_{1}\left(\pi_{1}\left(S^{1}\right)\right)$ is the quotient map where $i: S^{1} \rightarrow M$ is the inclusion induced by the $S^{1}$-action. Then for $x \in H\left(K\left(\pi^{\prime}, 1\right) ; \mathbb{Q}\right)$

$$
\Phi_{1}(M, f \alpha x)=\sum_{\lambda} \Phi_{1}\left(F_{\lambda} \circ F_{\lambda}, i_{\lambda} f \alpha x\right) \varepsilon^{d_{\lambda} / 4} .
$$

\section{§4. Vamishing Theorems}

Let $M$ be a closed connected spin manifold with an $S^{1}$-action, and $P$ a Spinstructure for $M$. The $S^{1}$-action is said to be of even type if it lifts to an action on 
$P$. Otherwise is said to be of odd type. Let $I$ be the element of order 2 in $S^{1}$. If the fixed point set $M^{I}$ of $I$ is not empty, then

$$
\operatorname{codim}\left(M^{I}\right)= \begin{cases}0(\bmod 4) & \text { if the action is even, } \\ 2(\bmod 4) & \text { if the action is odd }\end{cases}
$$

(see [1]). We get the following theorem by Theorem 3.2.

Theorem 4.1. Let $M$ be a closed connected spin manifold with an odd type $S^{1}$-action. Let $p: M \rightarrow M / S^{1}$ be the orbit map. Then for any $x \in H\left(M / S^{1} ; \mathbb{Q}\right)$,

$$
\Phi_{1}\left(M, p^{\prime} x\right)=0
$$

Proof. Let $I$ be the element of order 2. If $M^{I}=\phi$, the theorem is clear by Theorem 3.2.

In case $M^{I} \neq \phi$, let $F_{\lambda}$ be a connected component of $M^{I}$. Since the $S^{1}$-action is odd, $d_{\lambda}=\operatorname{codim} F_{\lambda} \equiv 2(\bmod 4)$. By Theorem 3.2 ,

$$
\Phi_{1}(M, p x)=\sum_{\lambda} \Phi_{1}\left(F_{\lambda} \circ F_{\lambda}, i_{\lambda} p x\right) \varepsilon^{d_{\lambda} / 4} .
$$

As we see in the proof of Theorem $3.2, \Phi_{1}(M, p x)$ equals the elliptic genus of some manifold up to a constant multiplication. So is $\Phi_{1}\left(F_{\lambda} \circ F_{\lambda}, i_{\lambda} p x\right)$. Hence they are polynomials in $\delta$ and $\varepsilon$ with coefficients in $\mathbb{Q}$. Since $d_{\lambda}-2 \equiv 0(\bmod 4)$, $\Sigma_{\lambda} \Phi_{1}\left(F_{\lambda} \circ F_{\lambda}, i_{\lambda} p x\right) \varepsilon^{\left(d_{\lambda}-2\right) / 4} \in \mathbb{Q}[\delta, \varepsilon]$. However $\left(\sum_{\lambda} \Phi_{1}\left(F_{\lambda} \circ F_{\lambda}, i_{\lambda} p x\right) \varepsilon^{\left(d_{\lambda}-2\right) / 4}\right) \varepsilon^{1 / 2}=$ $\Phi_{1}(M, p x) \in \mathbf{Q}[\delta, \varepsilon]$. Hence $\sum_{\lambda} \Phi_{1}\left(F_{\lambda} \circ F_{\lambda}, i_{\lambda} p x\right) \varepsilon^{\left(d_{\lambda}-2\right) / 4}=0$. As a result, $\Phi_{1}\left(M, p^{\prime} x\right)=0$.

For an $m$-dimensional closed oriented smooth manifold $M$ and $z \in H^{\prime}(M ; \mathbb{Q})$, we define

$$
\Phi_{2}(M, z)=\left\langle\hat{A}(M) \operatorname{ch}\left(\Theta_{q}(T(M)-[m]) \otimes \mathbb{C}\right) \cup z,[M]\right\rangle .
$$

Since $\delta$ and $\varepsilon$ are algebraically independent, we may replace $\Phi_{1}$ and $\varepsilon$ in Theorem 3.2 with $\Phi_{2}$ and $\tilde{\varepsilon}$ (in Theorem 2.1 (ii)). We denote the coefficient of $q^{\prime}$ in $\Theta_{q}(T(M))$ by $\Theta^{\prime}(T(M))$. Then we have

Theorem 4.2. Let $M$ be an m-dimensional closed spin manifold with an $S^{1}$-action, and let $p: M \rightarrow M / S^{1}$ be the orbit map. Suppose that $I$ is the element of order 2 in $S^{1}$. Then, for a non-negative integer $i$ with $i<\frac{\text { codim } M^{\prime}}{4}$ and for $x \in H\left(M / S^{1} ; \mathbf{Q}\right)$, we have

$$
\left\langle\hat{A}(M) \operatorname{ch}\left(\Theta^{\prime}(T(M)) \otimes \mathbb{C}\right) \cup p x,[M]\right\rangle=0 .
$$


Proof. Let $F_{\lambda}$ and $d_{\lambda}$ be those in Theorem 3.2. Then

$$
\Phi_{2}\left(M, p^{\prime} x\right)=\sum_{\lambda} \Phi_{2}\left(F_{\lambda} \circ F_{\lambda}, i_{\lambda}^{\dagger} p^{\prime} x\right) \tilde{\varepsilon}^{d_{\lambda} / 4} .
$$

Since the constant term of $\tilde{\varepsilon} \in \mathbb{Q}[[q]]$ is zero, the coefficient of $q^{\prime}$ in $\tilde{\varepsilon}^{d_{\lambda} / 4}$ is zero for $i<\frac{d_{\lambda}}{4}$. Therefore we have

$$
\left\langle\hat{A}(M) \operatorname{ch}\left(\Theta^{\prime}(T(M)-[m]) \otimes \mathbf{C}\right) \cup p^{\prime} x,[M]\right\rangle=0
$$

for $i<\frac{\operatorname{codim} M^{I}}{4}$. Since $\Theta^{\prime}(T(M))=\sum_{l=0}^{\prime} \Theta^{\prime}(T(M)-[m]) \Theta^{\prime-\prime}([m])$,

$$
\left\langle\hat{A}(M) \operatorname{ch}\left(\Theta^{\prime}(T(M)) \otimes \mathbf{C}\right) \cup p^{\prime} x,[M]\right\rangle=0
$$

for $i<\frac{\operatorname{codim} M^{I}}{4}$

We get the following corollaries from Theorems $4.1,4.2$ and [6, Theorem $1.1]$

Corollary 4.3. Let $M$ be a closed connected spin manifold with an odd type $S^{1}$-action. Let $f: M \rightarrow K(\pi, 1)$ and $\alpha: \pi \rightarrow \pi^{\prime}=\pi / f i_{1}\left(\pi_{1}\left(S^{1}\right)\right)$ be as in Corollary 3.3. Then for $x \in H\left(K\left(\pi^{\prime}, 1\right) ; \mathbf{Q}\right)$

$$
\Phi_{1}(M, f \alpha)=0 \quad(i=1,2) .
$$

Corollary 4.4. Let $M$ be a closed spin manifold with an $S^{1}$-action and $I$ the element of order 2 in $S^{1}$. Let $f: M \rightarrow K(\pi, 1)$ and $\alpha: \pi \rightarrow \pi^{\prime}=\pi / f_{1} i\left(\pi_{1}\left(S^{1}\right)\right)$ be as in Corollary 3.3. Then for a non-negative integer $k$ with $k<\frac{\text { codim } M^{\prime}}{4}$ and for $x \in H^{\prime}\left(K\left(\pi^{\prime}, 1\right) ; \mathbf{Q}\right)$

$$
\left\langle\hat{A}(M) \operatorname{ch}\left(\Theta^{h}(T(M)) \otimes \mathbf{C}\right) \cup f \alpha^{\prime} x,[M]\right\rangle,=0 .
$$

\section{§5. Higher Elliptic Genera of Level $N$}

In the following, $N$ is a fixed integer greater than 1 and the "variable" $x$ runs through the complex numbers. is the upper half-plane of the complex numbers, $\tau \in \mathfrak{h}$ and $q=e^{2 \pi i \tau}$. Let $L=2 \pi i(\mathbb{Z} \tau+\mathbb{Z})$ be a lattice and $\alpha=2 \pi i\left(\frac{h}{N} \tau+\frac{l}{N}\right)$ with $0 \leq k<N, 0 \leq l<N$ and $\alpha \neq 0$. In order to define the genus for stable almost complex manifolds, we introduce the function

$$
\Phi(x)=\left(1-e^{-1}\right) \prod_{n=1}^{\infty}\left(1-q^{n} e^{-1}\right)\left(1-q^{n} e^{1}\right) /\left(1-q^{n}\right)^{2}
$$


and we put

$$
f(x)=e^{(k / N) x} \Phi(x) \Phi(-\alpha) / \Phi(x-\alpha) .
$$

The function $f(x)$ is elliptic with respect to a sublattice $\tilde{L}$ of index $N$ in $L$ (see [7], [8]).

Let $M$ be a compact stable almost complex maifold and $c$ the total Chern class of $M$. If we write formally

$$
c=\prod_{t=1}^{d}\left(1+x_{t}\right),
$$

then the elliptic genus of level $N$ is defined as

$$
\varphi_{N}(M)=\left\langle\prod_{i=1}^{d} \frac{x_{t}}{f\left(x_{t}\right)},[M]\right\rangle .
$$

It is known that if $M$ has complex dimension $m, \varphi_{N}(M)$ is a modular form of weight $m$ on $\Gamma_{1}(N)=\left\{A \in S L_{2}(\mathbb{Z}) A \equiv\left(\begin{array}{ll}1 & 1 \\ 0 & 1\end{array}\right)(\bmod N)\right\}$.

We consider the case where $M$ has an $S^{1}$-action which preserves the stable almost complex structure. For each fixed point $p$, the circle acts in the stable tangent space $\tilde{T}_{p}$, hence integers $m_{1}, m_{2}, \ldots, m_{d}$ are defined such that $g \in S^{1}$ acts by the diagonal matrix $\left(g^{m_{1}}, g^{m_{2}}, \ldots, g^{m_{d}}\right)$. Let $v$ be an index for the connected components $\left(M^{S^{1}}\right)_{1}$, of the fixed point set $M^{S^{1}}$. The numbers $m_{1}, m_{2}, \ldots, m_{d}$ depend on $v$ and $m_{1}+m_{2}+\ldots+m_{d}$ also depends on $v$.

Definition. The $S^{1}$-action on $M$ is called $N$-balanced if for the components $\left(M^{S^{t}}\right)_{1}$ of the fixed point set the residue class of $m_{1}+m_{2}+\ldots+m_{d}$ modulo $N$ does not depend on $v$. If the action is $N$-balanced, the common residue class of $m_{1}+m_{2}+\ldots+m_{d}$ is called the type of the action and denoted by $t$.

In [8], Hirzebruch proved the following theorem.

Theorem 5.1 ([8])。 Let $M$ be a compact stable almost complex manifold with the first Chern class $c_{1} \equiv 0(\bmod N)$. If $M$ has an $S^{1}$-action which preserves the stable almost complex structure and the type $t$ of the action is $\not \equiv(\bmod N)$, then $\varphi_{N}(M)=0$.

We can consider generalized elliptic genera of level $N$ for a stable almost complex manifold in a similar way of previous sections. For a stable almost complex manifold $M$ with the total Chern class

$$
c(M)=\prod_{t=1}^{d}\left(1+x_{t}\right)
$$


and for $z \in H^{\prime}(M ; \mathbb{Q})$, we define

$$
\varphi_{N}(M, z)=\left\langle\prod_{l=1}^{d} \frac{x_{\imath}}{f\left(x_{l}\right)} \cup z,[M]\right\rangle .
$$

We can generalize Hirzebruch's theorem above as follows.

Theorem 5.2. Let $M$ be a compact stable almost complex manifold with the first Chern class $c_{1} \equiv 0(\bmod N)$. If $M$ has an $S^{1}$-action which preserves the stable almost complex structure and the type $t$ of the action is $\not \equiv 0(\bmod N)$, then for $x \in H^{\prime}\left(M / S^{1} ; \mathbb{Q}\right)$

$$
\varphi_{N}(M, p x)=0
$$

where $p: M \rightarrow M / S^{1}$ is the orbit map of the $S^{1}$-action.

Proof. As we saw in the proof of Theorem 3.2, for any $x \in H^{\prime}\left(M / S^{1} ; \mathbb{Q}\right)$, there exist a closed framed transverse $S^{1}$-submanifold $X$ of $M \times \mathbb{R}^{h}$ and $c \in \mathbb{Q}$ $\{0\}$ such that

$$
c p(x) \cap[M]=j[X]
$$

where $j: X \rightarrow M \times \mathbb{R}^{h}$ is the inclusion. Then, $c \varphi_{N}\left(M, p^{\prime} x\right)=\varphi_{N}(X)$.

Since $X$ is a framed submanifold of a stable almost complex manifold $M \times \mathbb{R}^{h}, X$ is also a stable almost complex manifold. If $c_{1}(M) \equiv 0(\bmod N)$, $c_{1}(X)=j c_{1}\left(M \times \mathbb{R}^{h}\right) \equiv 0(\bmod N)$. If the type of the action on $M$ is $\not \equiv(\bmod N)$, the type of the action on $M \times \mathbb{R}^{h}$ is $\not \equiv(\bmod N)$ and the type of the action on $X$ is also $\not \equiv 0(\bmod N)$. Hence $\varphi_{N}(X)=0$ from Theorem 5.1. As a result, $\varphi_{N}(M, p x)=0$.

From this theorem and [6, Theorem 1.1], we have

Corollary 5.3. Let $M$ be a compact stable almost complex manifold with the first Chern class $c_{1} \equiv 0(\bmod N)$. Suppose that $M$ has an $S^{1}$-action which preserves the stable almost complex structure and that the type tof the action is $\not \equiv 0(\bmod N)$. Let $f: M \rightarrow K(\pi, 1)$ and $\alpha: \pi \rightarrow \pi^{\prime}=\pi / f i\left(\pi_{1}\left(S^{1}\right)\right)$ be as in Corollary 3.3. Then for $x \in H\left(K\left(\pi^{\prime}, 1\right) ; \mathbb{Q}\right)$

$$
\varphi_{N}(M, f \alpha x)=0 \text {. }
$$

\section{Referemces}

[1] Atiyah, M.F. and Bott, R., A Lefschetz fixed point formula for elliptic complexes II. Applications, Ann. of Math., 88 (1968), 451-491.

[2] Atiyah, M. F. and Hirzebruch, F., Spin-manifolds and group actions, In Essays on Topology and Related Topics, Springer-Verlag, (1970), 18-28. 
[3] Bendersky, M., Applications of the Ochanine genus, Math. Z., 206 (1991), 443-455.

[4] Bott, R. and Taubes, C., On the rigidity theorems of Witten, J. Amer. Math. Soc., 2 (1989), 137186.

[5] Browder, W., $S^{\prime}$-actions on open manifolds, Contemporary Math., 37. (1982), 25-30.

[6] Browder, W. and Hsiang, W-C., G-actions and the fundamental group, Invent. Math., 65 (1982), 411-424.

[7] Hirzebruch, F., Berger, T. and Jung, R., Manifolds and Modular Forms, A Publication of the Max-Planck-Institut für Math., Bonn, 1992.

[8] Hirzebruch, F., Elliptic genera of level $N$ for complex manifolds, In [7] (Appendix III), 169-186.

[9] Hirzebruch, F. and Slodowy, P., Elliptic genera, involutions and homogeneous spin manifolds, Geom. Dedicata., 35 (1990), 309-343.

[10] Kawakubo, K., The Theory of Transformation Groups, Oxford Univ. Press, 1991.

[11] $\mathrm{Ku}, \mathrm{H}-\mathrm{T}$. and $\mathrm{Ku}, \mathrm{M}-\mathrm{C}$., The Pontrjagin numbers of an orbit map and generalized G-signature theorem, In Transformation Groups (Kawakubo, K., editor), Lect. Notes in Math., SpringerVerlag, 1375 (1989), 198-206.

[12] Liu, K.F., On mod 2 and higher elliptic genera, Comm. Math. Phys., 149 (1992), 71-95.

[13] Ochanine, S., Sur les genres multiplicatifs définis par des intégrales elliptiques, Topology, 26 (1987), 143-151.

[14] Zagier, D., Note on the Landweber-Stong elliptic genus, In Elliptic Curves and Modular Forms in Algebraic Topology (Landweber, P., editor), Lect. Notes in Math., Springer-Verlag, 1326 (1988), 216-224. 